\title{
Assessment of Prophylactic Intravenous Prochlorperazine at the End of Surgery for Prevention of Nausea and Vomiting During Intravenous Patient-Controlled Analgesia with Fentanyl
}

Hiromi Yoshinuma1 ${ }^{1}$, Mariko Kawana², Ryuichi Sekine ${ }^{3}$, Osamu Kobayashi ${ }^{1}$

Department of Anesthesiology ${ }^{1}$, Pharmacy ${ }^{2}$, and Palliative Care ${ }^{3}$,Kameda Medical Center, Kamogawa, Chiba, Japan

\section{Background}

- Addition of droperidol to patient-controlled analgesia (PCA) is the current gold standard prophylactic anti-emetic in fentanyl-associated postoperative nausea and/or vomiting (N/V)

- Even when droperidol is added to PCA, some patients complain of N/V

\section{Study Goal}

- To assess whether prophylactic intravenous prochlorperazine (PCZ) at the end of surgery could prevent fentanyl-associated N/V during fentanyl PCA

\section{Material and Methods}

- Retrospective cohort study from February to August 2016

- PCA prepared with fentanyl $15 \mathrm{mcg} / \mathrm{ml}$ and droperidol $40 \mathrm{mcg} / \mathrm{ml}$ at $1 \mathrm{ml} /$ hour plus a $1 \mathrm{ml}$ bolus and a lock-out period of $10 \mathrm{~min}$

- A total of 74 patients received PCA immediately after surgery

39 patients received a single dose of PCZ $5 \mathrm{mg}$ immediately after surgery (Group A)

35 patients did not (Group B)

- All patients were followed for the first 24 hours and could request a single dose of PCZ whenever they experienced N/V

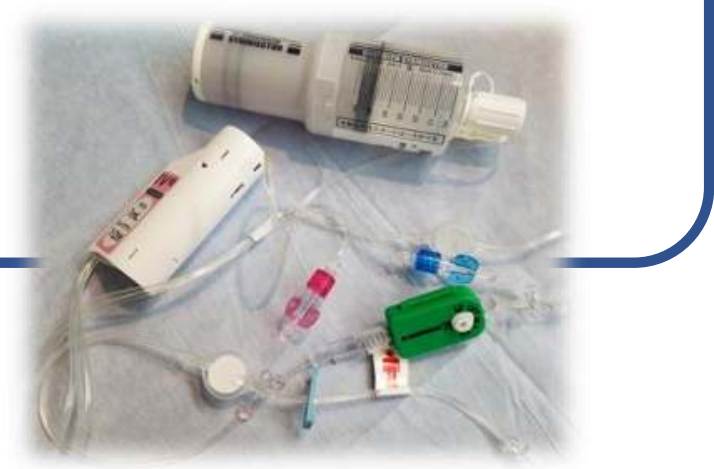

PCA pump (COOPDECH syrinjector PCA device, Daiken-iki, Japan)

\section{Outcome Measures}

- Number of patients requesting PCZ documented during two time periods: $0-12$ hours and $>12-24$ hours after surgery

- Number of times PCZ was requested during the two time periods

\section{Statistical analysis}

- Chi-squared test and Student's t-test evaluated the difference between the two groups

- $P<0.05$ was considered significant

\section{Results}

Table 1. Baseline Characteristics of Patients Stratified by Group, N=74

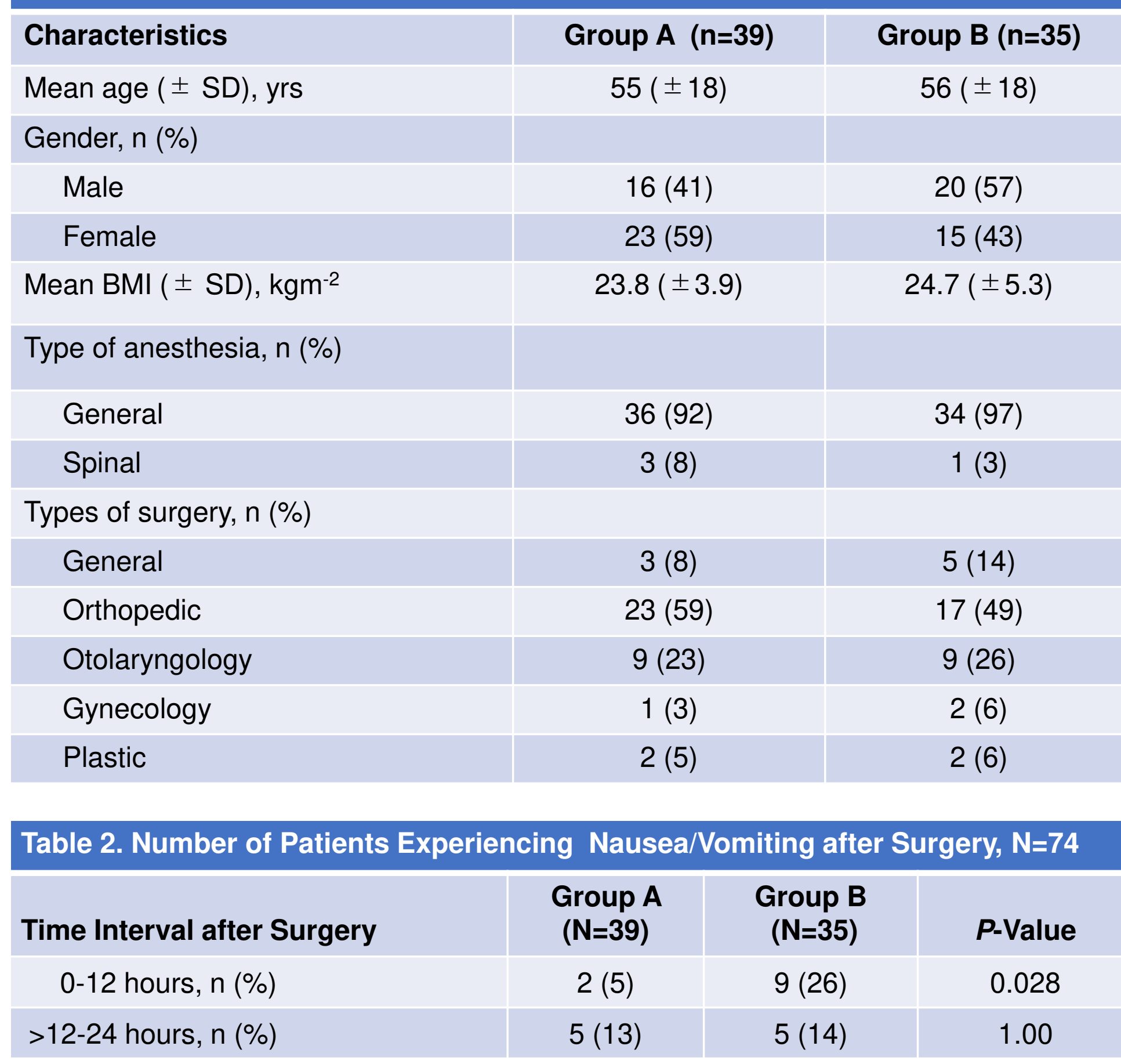

Table 3. Number of Patients and Times Prochlorperazine Requested after Surgery

\begin{tabular}{|c|c|c|c|c|c|c|}
\hline \multirow{3}{*}{$\begin{array}{l}\text { Number of Times } \\
\text { Prochlorperazine } \\
\text { Requested after } \\
\text { Surgery }\end{array}$} & \multicolumn{6}{|c|}{ Number of Patients requesting Prochlorperazine, $\mathbf{n}(\%)$} \\
\hline & \multicolumn{2}{|c|}{$\begin{array}{c}\text { 0-12 Hours } \\
\text { After Surgery }\end{array}$} & \multirow[t]{2}{*}{$P$-value } & \multicolumn{2}{|c|}{$\begin{array}{l}>12-24 \text { Hours } \\
\text { After Surgery }\end{array}$} & \multirow[t]{2}{*}{$P$-value } \\
\hline & Group A & Group B & & Group A & Group B & \\
\hline \multicolumn{7}{|l|}{ None } \\
\hline With N/V & $0(0)$ & $1(3)$ & & $3(8)$ & $2(6)$ & \\
\hline Without N/V & 37 (95) & $26(74)$ & & $34(87)$ & $30(86)$ & \\
\hline \multicolumn{7}{|l|}{ Once } \\
\hline With N/V & $2(5)$ & $5(14)$ & & $1(3)$ & $2(6)$ & \\
\hline Without N/V & $0(0)$ & $0(0)$ & & $0(0)$ & $0(0)$ & \\
\hline \multicolumn{7}{|l|}{ Twice } \\
\hline With N/V & $0(0)$ & $3(9)$ & & $1(3)$ & $1(3)$ & \\
\hline \multirow[t]{3}{*}{ Without N/V } & $0(0)$ & $0(0)$ & & $\begin{array}{c}0.08 \\
(0.35)\end{array}$ & $0(0)$ & \\
\hline & \multicolumn{6}{|c|}{ Mean Number of Times Prochlorperazine Requested ( \pm SD) } \\
\hline & $\begin{array}{c}0.05 \\
(0.22)\end{array}$ & $\begin{array}{c}0.31 \\
(0.62)\end{array}$ & 0.02 & $\begin{array}{c}0.08 \\
(0.35)\end{array}$ & $\begin{array}{c}0.11 \\
(0.40)\end{array}$ & 0.67 \\
\hline \multicolumn{7}{|c|}{${ }^{*} \mathrm{PCZ}$ means prochlorperazine; ${ }^{\dagger} \mathrm{N} / \mathrm{V}$ means nausea and/or vomiting; } \\
\hline
\end{tabular}

Conclusion

- Our experience suggests that prophylactic intravenous PCZ at the end of surgery may be effective in preventing N/V during fentanyl PCA use in the first 12 hours after surgery 